\title{
Prominent Hemophagocytosis in Cerebrospinal Fluid in A Patient With Bacterial Meningitis
}

\author{
Alauldeen Alqasim ${ }^{1}$ and Rajeev Kariyattil ${ }^{2}$ \\ ${ }^{1}$ Sultan Qaboos University Hospital \\ ${ }^{2}$ Affiliation not available
}

May 18, 2020

\begin{abstract}
A 39 year old patient was presented with bacterial meningitis after having endoscopic surgery for removal of a benign pituitary tumor. Cerebrospinal cytology showed a marked hemophagocytic activity.
\end{abstract}

Title: Prominent Hemophagocytosis in Cerebrospinal Fluid in a Patient with Pneumococcal Meningitis

Authors: Alauldeen Mudhafar Zubair Alqasim ${ }^{1}$, Rajeev Kariyattil ${ }^{2}$

Affiliations:

1 Oman, Muscat, Sultan Qaboos University Hospital, Department of Hematology,

2 Oman, Muscat, Sultan Qaboos University Hospital, Department of Surgery

\section{Corresponding author:}

Dr. Alauldeen Alqasim

Email: aladdinalqasim@yahoo.com

Abstract: Lumbar puncture is a common medical procedure especially in neurology and hematology patients. It is done mainly for the diagnosis of meningitis, SAH, CNS leukemia and other neurological disorders. Analysis usually includes biochemistry, Gram stain, culture, cell count and morphology.

Key words: Hemophagocytosis, meningitis, CSF

Key Clinical Message: Hemophagocytosis is usually observed in bone marrow. However, it can be an incidental finding in other body fluids in severe infections like cerebrospinal fluid in case of meningitis. This should alert the clinicians to rule out primary type of hemophagocytic syndrome.

Case: A 39 year old patient had endoscopic trans-sphenoidal drainage of a supra-sellar benign cystic lesion. Five weeks later, she was presented with bacterial meningitis complicated by cavernous sinus thrombosis. CSF analysis revealed negative viral screen, Gram positive cocci and bacterial antigen test was positive for strptococcus pnumoniae. Leukocytes $=810 / \operatorname{cumm}(\mathrm{NR}=0-10)$ and $\mathrm{RBC}=2000 / \operatorname{cumm}(\mathrm{NR}=0)$. CSF cytology showed a very large number of neutrophils in addition to many monocytes and few plasma cells. What was striking is the prominent hemophagocytic activity (Figures 1-4). This is usually observed in BM aspirate of patients with primary or secondary HLH but a very unusual phenomenon in CSF. This finding should alert the physicians to exclude an underlying primary HLH. Her CBC results showed $\mathrm{Hb}=11 \mathrm{~g} / \mathrm{l}$, leukocytes $=22.6^{*} 10^{9} / 1$ (neutrophils $\left.=20.1 * 10^{9} / \mathrm{l}\right)$, plt $=342^{*} 10^{9} / \mathrm{l}$. Fibrinogen $=5.4 \mathrm{~g} / \mathrm{l}(\mathrm{NR}=1.7-3.6 \mathrm{~g} / \mathrm{l})$. 
Meningococcal sepsis secondary to an underlying primary HLH has been reported. ${ }^{1}$ Another study of 96 cases of primary HLH found that CSF examinations showed abnormalities in 23 patients (39.0\%), of which 10 patients had elevated white blood cell counts and 13 patients had elevated protein contents. ${ }^{2}$ The patient did not fulfil the criteria of primary HLH.

Informed consent: The patient's next of kin signed written informed consent.

Conflict of interests: None declared.

Authors' contribution: AMZ Alqasim wrote the draft and provided images, R Kariyattil provided clinical care for the patient.

\section{Abbreviations:}

CBC: Complete Blood Count

CNS: central nervous system

CSF: cerebrospinal fluid

HLH: hemophagocytic lymphohistiocytosis

NR: normal range

SAH: Subarachnoid Hemorrhage

Word count: 186

\section{References:}

1. Bentur H, Horan M, Kerr S. Meningococcal Sepsis and Haemophagocytic Lymphohistiocytosis. Arch Dis Child 2012; 97(Suppl 1):A1-A186.

2. Song Y, Pei RJ, Wang YN, Zhang J, Wang Z. Central Nervous System Involvement in Hemophagocytic Lymphohistiocytosis in Adults: A Retrospective Analysis of 96 Patients in a Single Center. Chin Med J (Engl). 2018;131(7):776-783. doi:10.4103/0366-6999.228234

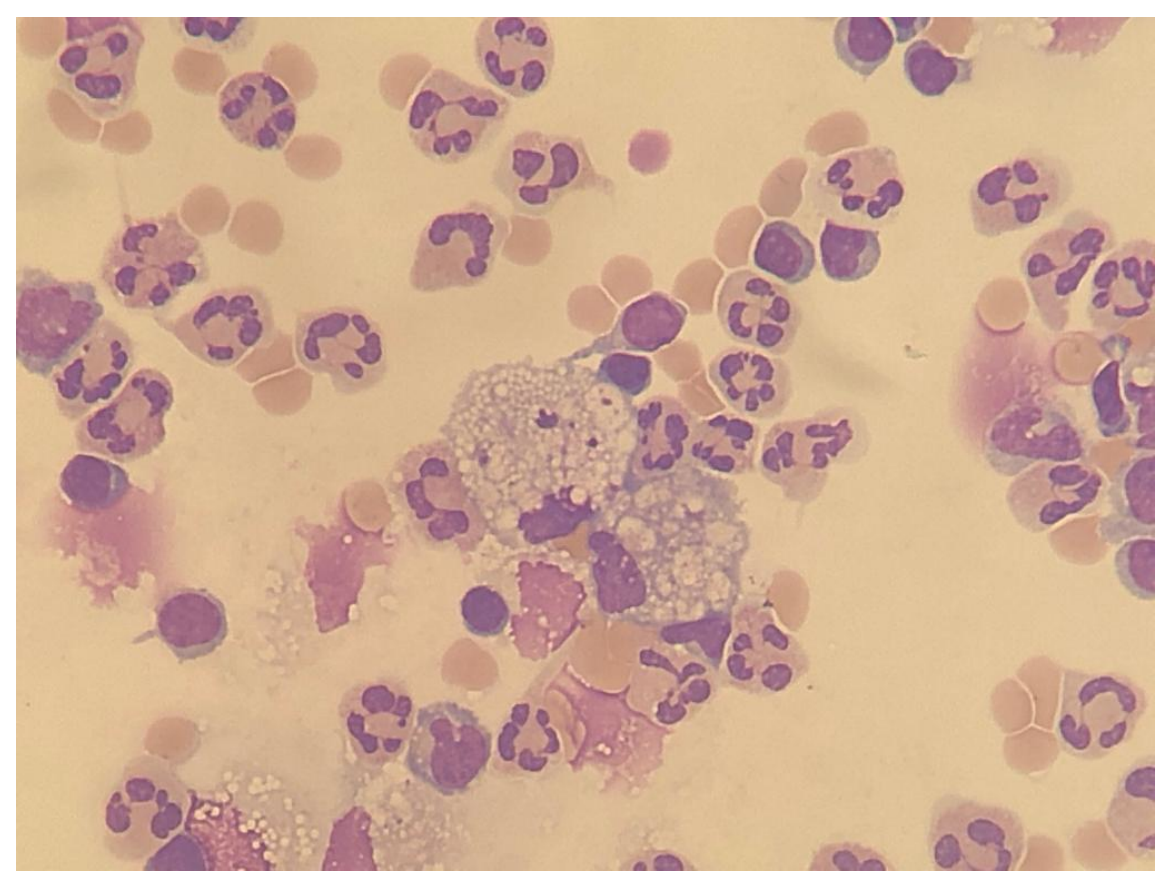



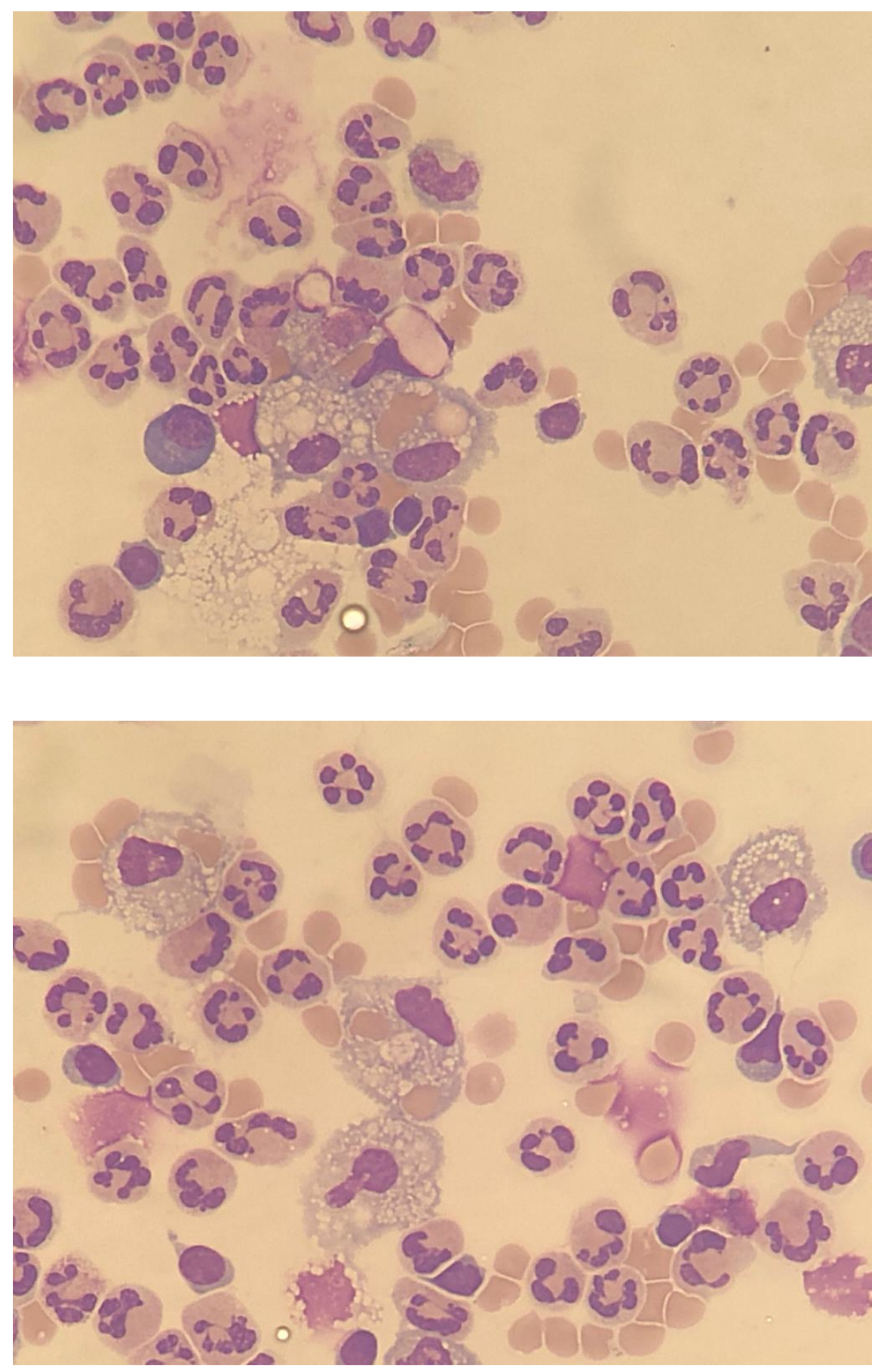


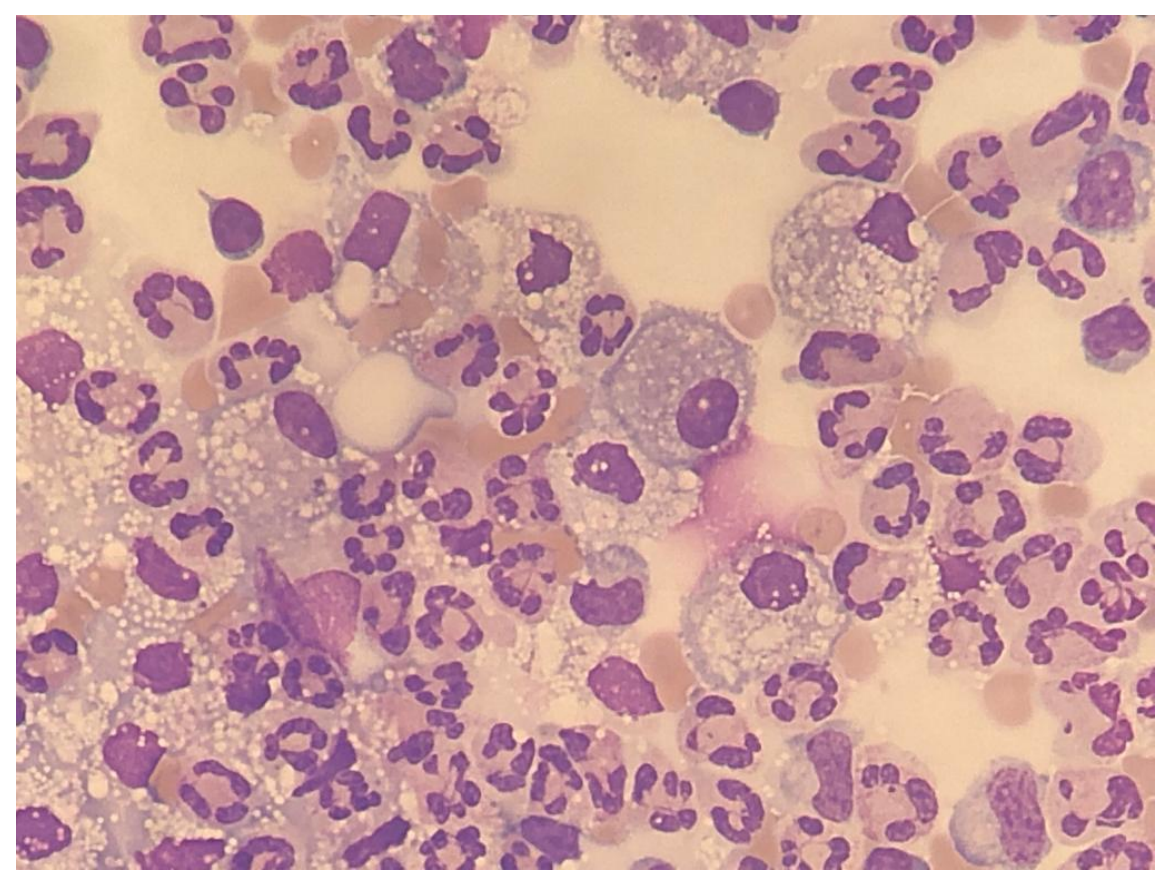

